PROCEEDINGS OF THE AMERICAN MATHEMATICAL SOCIETY

Volume 33, Number 2, June 1972

\title{
RINGS WHOSE QUASI-INJECTIVE MODULES ARE INJECTIVE
}

\author{
K. A. BYRD
}

\begin{abstract}
A ring $R$ is called a V-ring, respectively SSI-ring, respectively QII-ring if simple, respectively semisimple, respectively quasi-injective, right $R$-modules are injective. We show that $R$ is SSI if and only if $R$ is a right noetherian V-ring and that any SSI-ring is a finite ring direct sum of simple SSI-rings. We show that if $R$ is left noetherian and SSI then $R$ is QII provided $R$ is hereditary and that in order for $R$ to be hereditary it suffices that maximal right ideals of $R$ be reflexive. An example of Cozzens is cited to show these rings need not be artinian.
\end{abstract}

The rings $R$ which have the property that quasi-injestive right $R$ modules are injective (herein called QII-rings) have been little mentioned in the literature though related classes of rings have been extensively examined. Consider the classes of $R$-modules consisting of (1) all $R$ modules, (2) injective $R$-modules, (3) projective $R$-modules, (4) quasiinjective $R$-modules, and (5) quasi-projective $R$-modules. Of all the nontrivial inclusions one can assume between two of these classes, all but one has been shown to characterize the ring $R$ as either semisimple artinian, quasi-Frobenius, or uniserial. That one excepted is the class of QII-rings whose internal structure, it seems, has not been completely determined.

This paper does not attempt a general structure theorem but investigates the connection between QII-rings and rings whose semisimple right modules are injective (SSI-rings). Clearly every QII-ring is an SSI-ring. We show that SSI-rings are the same as right noetherian, V-rings and that an SSI-ring is a finite ring direct sum of simple SSI-rings. Finally, it is shown that a left noetherian, right SSI-ring which is hereditary is a QII-ring and that for such a ring to be hereditary it suffices that maximal right ideals be reflexive.

All rings are assumed to be associative rings with identity and all modules are unital, right modules unless otherwise indicated. V, SSI, and QII refer to the appropriate condition on the right modules of the ring.

Received by the editors July 6, 1971.

AMS 1970 subject classifications. Primary 16A52, 16A62; Secondary 16A46.

Key words and phrases. Quasi-injective module, V-ring, Morita-equivalent, quotient ring.

(c) American Mathematical Socieiy 1972 
1. V-rings and SSI-rings. A ring $R$ is called a (right) $V$-ring if every simple, right $R$-module is injective. $R$ is called an SSI-ring if every semisimple, right $R$-module is injective.

The following theorem is generally attributed to Villamayor.

THEOREM (VILLAMAYOR). The following are equivalent:

(1) $R$ is a V-ring.

(2) Every right ideal of $R$ is the intersection of the maximal right ideals which contain it.

(3) For every right $R$-module $M, \operatorname{Rad} M=0$.

Proof. (1) implies (3). Let $M$ be a nonzero right $R$-module. If $0 \neq x \in M$, let $K_{x}$ be a maximal along the submodules of $M$ which exclude $x$. Then $M / K_{x}$ is uniform since every nonzero submodule contains the module $\left(x R+K_{x}\right) / K_{x}$ generated by $x+K_{x}$. Since $\left(x R+K_{x}\right) / K_{x}$ is simple, it is injective and a direct summand of $M / K_{x}$. Thus $M / K_{x}=\left(x R+K_{x}\right) / K_{x}$ and $K_{x}$ is a maximal submodule of $M$. It follows that $x \notin \operatorname{Rad} M$. Thus $\operatorname{Rad} M=0$.

(3) implies (2). Given a right ideal $I, \operatorname{Rad} R / I=0$.

(2) implies (1). Let $S$ be simple, $I$ a right ideal of $R$ and $\phi$ an $R$-homomorphism of $I$ into $S$. We need to extend $\phi$ to a mapping of $R$ into $S$. We may assume $\phi$ is epi and replace $\phi$ by the canonical epimorphism $\pi^{\prime}: I \rightarrow I / K, \pi^{\prime}(i)=i+K$, where $K=\operatorname{Ker} \phi, I / K \cong S$. It suffices to extend $\pi^{\prime}$ to $R$. (2) implies $\operatorname{Rad} R / K=0$ and since $I / K$ is simple there is a maximal submodule $M / K$ of $R / K$ such that $I / K \oplus M / K=R / K$. The canonical epimorphism $\pi: R \rightarrow R / K$ composed with the projection onto $I / K$ then extends $\pi^{\prime}$.

Note that any V-ring is Jacobson semisimple and hence semiprime.

Proposition 1. $R$ is an SSI-ring if and only if $R$ is a right noetherian $V$-ring.

Proof. A right noetherian V-ring is clearly an SSI-ring.

Let $R$ be an SSI-ring and $I_{0} \subset I_{1} \subset I_{2} \subset \cdots$ be a strictly ascending sequence of right ideals. Since $R$ is a V-ring we can find right ideals $M_{k}$ such that $I_{k-1} \subseteq M_{k} \subset I_{k}$ and $I_{k} / M_{k}$ is simple. Let $I=\bigcup I_{k}$. The canonical epimorphisms $I \rightarrow I / M_{k}$ together with the projections $I / M_{k} \rightarrow I_{k} / M_{k}$ (which exist since $I_{k} / M_{k}$ is a direct summand of $I / M_{k}$ ) compose to give epimorphisms $\pi_{k}: I \rightarrow I_{k} / M_{k}$ such that if $x \in I_{j}$ then $\pi_{k}(x)=0$ for $k>j$. Thus the $\pi_{k}$ 's induce a map, $\pi: I \rightarrow \amalg I_{k} / M_{k}$, of $I$ into the coproduct of the $I_{k} / M_{k}$ 's. Extending $\pi$ to a map of $R$ into $\amalg I_{k} / M_{k}$ we see that $\operatorname{Im} \pi$ is contained in $\bigcup_{k=1}^{n} I_{k} / M_{k}$ for some integer $n$. Since $\pi_{k}(x) \neq 0$ for $x \in I_{k}-M_{k}$ we see that our sequence has length $\leqq n$. (This argument is adapted from [5].)

COROLlaRY. A commutative SSI-ring is semisimple artinian. 
Proof. Kaplansky has shown that a commutative ring is a V-ring if and only if it is regular. It is well known that a noetherian, regular ring is semisimple artinian.

COROLLARY. If $R$ is a $V$-ring then any artinian $R$-module is noetherian. If $R$ is an SSI-ring then an $R$-module is artinian if and only if it is semisimple of finite length.

Proof. If $R$ is a V-ring and $M$ is a nonzero $R$-module we can find a maximal proper submodule $M_{1}$. Repeating this process we generate a chain $M \supset M_{1} \supset M_{2} \supset \cdots$ which terminates, if $M$ is artinian, in a composition series for $M$.

The second statement is immediate since if $R$ is SSI and $M$ is artinian then its socle, Soc $M$, is injective and essential in $M$. So $M=\operatorname{Soc} M$.

An $R$-module $M$ is said to be divisible if $M r=M$ for every regular element $r$ of $R$. Injective modules are divisible.

A ring $R$ is said to be right Goldie if $R$ has ascending chain condition on both complement right ideals and annihilator right ideals.

THEOREM (FAITH). If $R$ is a prime, right Goldie $V$-ring then $R$ is simple.

Proof. Goldie [4] proved that an essential right ideal of a semiprime right Goldie ring contains a regular element. If $I$ is a nonzero two-sided ideal then for any nonzero right ideal $K$ of $R$ we have that since $R$ is prime $0 \neq K I \subseteq I \cap K$. Thus $I$ is essential. $I$ cannot be proper since, if so, we could find a maximal right ideal $M \supseteq I$. Thus taking $r$ regular in $I$, $(R / M) r=0$, so $R / M$ is not divisible and $R$ is not a V-ring.

It is not hard to see that Faith's result means that, in a right Goldie V-ring, prime ideals are the same as maximal ideals.

Proposition 2. Any right Goldie V-ring is a finite ring direct sum of simple rings.

Proof. It is well known that if $R$ is a semiprime, right Goldie ring then the set $S$ of maximal annihilator ideals of two-sided ideals of $R$ is a finite set of prime ideals with zero intersection. Each of these ideals is maximal by Faith's result. Taking $\left\{M_{i} \mid i=1, \cdots, n\right\}$ to be a subset of $S$ with irredundant zero intersection the usual map $R \rightarrow \prod_{i=1}^{n} R / M_{i}, r \mapsto\left(r+M_{1}\right.$, $r+M_{2}, \cdots, r+M_{n}$ ) is a ring isomorphism. (It is epi since $R=M_{i}+$ $\bigcap_{j \neq i} M_{j}$ for each $i$.)

There are nonartinian SSI-rings. In [3] Cozzens has given a simple, noncommutative domain which has all its one-sided ideals principal and is a V-ring but not a division ring. Cozzens' example is a nonartinian SSI-ring. Cozzens' ring is also QII (see [2]) and, in fact, both left and right QII. 
2. Noetherian hereditary V-rings are $\mathbf{Q I}$. In the following $R$ is assumed to be left and right noetherian. Then "left hereditary" and "right hereditary" are equivalent conditions and we simply use the term "hereditary."

If $R$ is a right Goldie semiprime ring then every $R$-module $M$ has a torsion submodule $M_{t}$ where $M_{t}=\{m \in M \mid m r=0$ for some regular $r \in R\}=$ $\{m \in M \mid m I=0$ for some essential right ideal $I\}$. The torsion submodule is, in this case, the same as the right singular submodule of $\mathrm{R}$. E. Johnson. If $M$ is torsion-free, i.e. $M_{t}=0$, and divisible then one can extend the $R$-module structure of $M$ to make $M$ a $Q$-module where $Q$ is the right classical quotient ring of $R$. For since $M$ is divisible then given $m \in M$ and $b$ regular in $R$ there is $m^{\prime} \in M$ such that $m=m^{\prime} b$ and since $M_{t}=0, m^{\prime}$ is unique. Letting $m^{\prime}=m b^{-1}$ defines the $Q$-module structure on $M$. The following lemma is well known.

Lemma. If $R$ is semiprime, right Goldie and $M$ is torsion-free and divisible then $M$ is injective.

Proof. Given an $R$-homomorphism $\phi: I \rightarrow M$ where $I$ is a right ideal of $R$ we extend $\phi$ to a mapping of $I Q$ into $M$ by defining $\phi_{1}\left(i b^{-1}\right)=$ $\phi(i) b^{-1}$ for $i \in I$ and $b$ regular in $R$. Since $I Q$ is a direct summand of $Q$ we can extend $\phi_{1}$ to a map $\phi_{2}$ of $Q$ into $M$. The restriction of $\phi_{2}$ to $R$ is the desired extension of $\phi$.

Recall that if $M$ is a right $R$-module then $M^{*}=\operatorname{Hom}_{R}(M, R)$ is a left $R$-module and there is a natural $R$-homomorphism $\delta_{M}: M \rightarrow M^{* *}=$ $\operatorname{Hom}_{R}\left(M^{*}, R\right)$ given by $m^{*} \delta_{M I}(m)=m^{*} m$ if $m \in M$ and $m^{*} \in M^{*} . M$ is called reflexive when $\delta_{M I}$ is an isomorphism [1].

If $R$ is a right Goldie, semiprime ring, and $K$ is a right ideal of $R$ and if $k^{*} \in K^{*}$ then thinking of $k^{*}$ as a mapping of $K$ in $Q$, the right quotient ring, since $Q_{R}$ is injective there is a $q \in Q$ so that $k^{*} k=q k$ for all $k \in K$. If $K$ is essential then, since $Q$ is torsion-free, this establishes an isomorphism $K^{*} \cong\{q \in Q \mid q K \subseteq R\}$.

Proposition 3. If $R$ is a left and right noetherian $V$-ring then the following are equivalent:

(1) $R$ is hereditary.

(2) Maximal right ideals are reflexive.

(3) If I is an essential right ideal then $R / I$ is artinian.

(4) Every divisible $R$-module is injective.

Proof. Note if $K$ is an essential right ideal we can identify

$$
K^{*}=\{q \in Q \mid q K \subseteq R\} \text { and } K^{* *}=\left\{q \in Q \mid K^{*} q \subseteq R\right\}
$$

where $Q$ is the left and right quotient ring of $R$. 
(1) implies (2). (1) implies that right ideals are projective and it is well known that finitely generated projective modules are reflexive.

(2) implies (3). Let $I_{0} \supset I_{1} \supset I_{2} \supset \cdots$ be a strictly descending sequence of right ideals each containing the essential right ideal $I$. Then $I_{j}^{*} \subseteq I_{j+1}^{*}$. Let $x \in I_{j}-I_{j+1}$. Since $R$ is a V-ring there is a maximal right ideal $M \supseteq I_{j+1}$ and such that $x \notin M$. If $I_{j}^{*}=I_{j+1}^{*}$ then $x \in I_{j} \subseteq I_{j}^{* *}=I_{j+1}^{* *} \subseteq M^{* *}=M$, a contradiction. Thus we have always $I_{j}^{*} \subset I_{j+1}^{*}$. If $r$ is a regular element of $I$ then $r R \subset I$ so $(r R)^{*}=R r^{-1} \supseteq I^{*} \supseteq I_{j}^{*}$ for all $j$. So $I_{0}^{*} \subset I_{1}^{*} \subset \cdots$ is a strictly ascending sequence of $R$-submodules of the noetherian module $R r^{-1}$. It must be finite and then $I_{0} \supset I_{1} \supset \cdots$ is finite. It follows that $R / I$ is artinian when $I$ is an essential right ideal.

(3) implies (4). Let $M$ be a nonzero torsion module, i.e. $M=M_{t}$. Then if $m \in M, m R \cong R / I$ where $I$ is the annihilator right ideal of $m$, and $I$ is essential. $m R$ is thus artinian and therefore semisimple since $R$ is SSI. Since $M=\sum_{m \in M} m R, M$ is semisimple. Thus for every $R$-module $M$, $M_{t}$ is an injective, semisimple module. So $M=M_{t} \oplus M_{0}$ for some submodule $M_{0}$ which is torsion-free. If $M$ is divisible so is $M_{0} \cong M / M_{t}$. Then by the lemma, $M_{0}$ is injective and then so is $M$.

(4) implies (1). Since divisibility is equivalent to injectivity and factor modules of divisible modules are always divisible then factor modules of injective modules are injective.

The proof "(2) implies (3)" owes a great deal to Webber [7] who proved (1) implies (3) without assuming $R$ is a V-ring.

Note that $M_{t} \subseteq \operatorname{Soc} M$ always and $M_{t}=\operatorname{Soc} M$ if and only if Soc $R=0$ which is the only interesting case anyway.

Proposition 4. A left and right noetherian hereditary $V$-ring $R$ is a QII-ring.

Proof. By Proposition 1 we may assume $R$ is a simple ring. For any right ideal $K$ of $R, K \subseteq K^{*} K \subseteq R$ and $K^{*} K$ is a two-sided ideal of $R$. Thus for any nonzero right ideal $K$ we have $K^{*} K=R$. Thus any nonzero right ideal $K$ is a generator of the category $\operatorname{Mod} R$ of right $R$-modules. Since $K$ is finitely generated and projective it is a progenerator and it follows that if $S=\operatorname{End}_{R} K$ is the ring of $R$-endomorphisms of $K$ then $\operatorname{Mod} R$ is Morita equivalent to Mod $S$. Thus the proposition is true for $R$ if and only if it is true for some $S=\operatorname{End}_{R} K, K$ a nonzero right ideal.

Taking $K$ to be a uniform right ideal we see that we may assume $R$ is an Ore domain. By Proposition 3, (3) $R / I$ is semisimple unless $I=0$. From [6] we see that the indecomposable injective $R$-modules are either simple or isomorphic to $Q_{R}$, the injective hull of $R_{R}$. $Q$ is the right and left quotient ring of $R$ and is a division ring. If $M$ is a quasi-injective $R$ module then it is a direct sum of indecomposable quasi-injectives, $M=$ $\amalg_{j \in J} M_{j}$, where the injective hull of $M_{j}$ is either simple (in which case 
it is $M_{j}$ ) or $Q_{R}$. But $Q_{R}$ has no proper quasi-injective submodule since such a module would be a $Q-R$ submodule of the $Q-R$ bimodule $Q$, i.e. a left ideal of $Q$. Thus $M$ is a coproduct of modules each either simple or isomorphic to $Q_{R}$, so $M$ is injective.

COROLlary. If $R$ is a left noetherian V-ring whose right ideals are principal then $R$ is a $Q I I-$ ring.

Proof. Note if Soc $R \neq 0$ then since $R$ is semiprime and Soc $R$ is injective as an $R$-module then Soc $R$ is a two-sided ideal direct summand of $R$. So $R$ is the direct product of a semisimple artinian ring and a ring with zero socle. Thus we may consider $R$ to have zero socle.

Since maximal right ideals are principal and essential it follows that each maximal right ideal is generated by a regular element of $R$ and is therefore free. Then certainly maximal right ideals are reflexive. The corollary now follows from Proposition 3 and Proposition 4.

It should be made clear that the author is unaware of any examples of nonartinian SSI-rings other than those of Cozzens [3] and, of course, those Morita-equivalent to such rings. Thus I have no example of an SSI-ring which is not also left noetherian and hereditary.

\section{REFERENCES}

1. H. Bass, Finitistic dimension and a homological generalization of semi-primary rings, Trans. Amer. Math. Soc. 95 (1960), 466-488. MR 28 \#1212.

2. K. A. Byrd, When are quasi-injectives injective?, Canad. Math. Bull. (to appear).

3. J. H. Cozzens, Homological properties of the ring of differential polynomials, Bull. Amer. Math. Soc. 76 (1970), 75-79, MR 41 \#3531.

4. A. W. Goldie, Semi-prime rings with maximum condition, Proc. London Math. Soc. (3) 10 (1960), 201-220. MR 22 \#2627.

5. R. P. Kurshan, Rings whose cyclic modules have finitely generated socle, J. Algebra 15 (1970), 376-386. MR 41 \#5403.

6. E. Matlis, Injective modules over Noetherian rings, Pacific J. Math. 8 (1958), 511-528. MR 20 \#5800.

7. D. B. Webber, Ideals and modules of simple Noetherian hereditary rings, J. Algebra 16 (1970), 239-242.

Department of Mathematics, University of North Carolina at Greensboro, Greensboro, North Carolina 27412 